\title{
ON THE PARTICULAR SOLUTION OF THE NONHOMOGENEOUS RIEMANN PROBLEM IN THE WEIGHTED SMIRNOV CLASSES WITH THE GENERAL WEIGHT
}

\author{
SABINA R. SADIGOVA
}

\begin{abstract}
In this work the nonhomogeneous Riemann problem of the theory of analytic functions with a piecewise Hölder coefficient in weighted Smirnov classes with a general weight is considered. The sufficient conditions on the coefficient of the problem and on the weight function are found, so that the considered Cauchy type integral has been a particular solution to this problem.
\end{abstract}

\section{Introduction}

The theory of Riemann boundary value problems has a deep and long history. Subsequently, in connection with applications in various fields of mathematics, this theory is developed in the works of various mathematicians (see, for example, $[34,18,19,11,30,33,28,14,37,38,39,17,23])$. Riemann-theory of Riemann problems was developed in a monograph [14]. The weighted case with power weight in various statements was studied in [25, 24]. The Riemann boundary value problems generated by the metrics of the Lebesgue space with a variable summability exponent and the Morrey space were studied in $[22,29,9,8]$. More detailed information in this direction can be found in [35], in which the solvability of the homogeneous Riemann problem with a piecewise continuous coefficient in weighted Smirnov classes with a weight of the general form is studied. It should be noted that the methods of this theory are used in other areas of mathematics such as approximation theory, spectral theory of differential operators, and others. Relative to the concerning problems see $[31,32,26,27,2,3,4,5,6,7]$.

This work deals with the nonhomogeneous Riemann problem of the theory of analytic functions with a piecewise Hölder coefficient in weighted Smirnov classes with a general weight. The sufficient conditions on the coefficient of the problem and on the weight function are found in order to the considered Cauchy type integral be a particular solution of this problem.

It should be noted that earlier in papers $[35,36]$ a general solution was constructed of the homogeneous Riemann problem with a piecewise-continuous coefficient in weighted Smirnov classes with a general weight.

2010 Mathematics Subject Classification. 30E25, 30D55, 32A55.

Key words and phrases. Riemann problem, Smirnov classes, weight function. 
In [36] the solvability of the nonhomogeneous Riemann problem with a piecewise continuous coefficient is studied in weighted Smirnov classes in case the weight has a power form.

\section{Necessary information}

In this section, we state some notations and facts to be used to obtain our results. By $O_{r}\left(z_{0}\right)$ we denote a circle of radius $r$ centered at $z_{0}$ on the complex plane $\mathbb{C}$, i.e. $O_{r}\left(z_{0}\right) \equiv\left\{z \in \mathbb{C}:\left|z-z_{0}\right|<r\right\} .|M|$ means the Lebesgue (linear) measure of the set $M \subset \Gamma$ where $\Gamma \subset \mathbb{C}$ is some rectifiable curve. Notation $f(x) \sim g(x), x \in M$, means $\exists \delta>0: 0<\delta \leq\left|\frac{f(x)}{g(x)}\right| \leq \delta^{-1}, \forall x \in M$.

Let's give a definition for Carleson curve.

Definition 2.1. Jordan rectifiable curve $\Gamma$ on the complex plane is called Carleson curve or regular curve if

$$
\sup _{z \in \Gamma}\left|\Gamma \cap O_{r}(z)\right| \leq c r, \quad \forall r>0,
$$

where $c$ is a constant independent of $r$.

For more information about this concept see, e.g., [12, 16, 15].

Let $\Gamma$ be some Jordan rectifiable curve in the complex plane $\mathbb{C}$. A measurable function $\omega: \Gamma \rightarrow[0, \infty]$ is said to be a weight if $\omega^{-1}(\{0, \infty\})$ has measure zero.

Definition 2.2. We will say that the weight function $\omega: \Gamma \rightarrow R^{+}=(0,+\infty)$ belongs to the Muckenhoupt class $A_{p}(\Gamma)(p>1)$, if

$$
\operatorname{supsup}_{z \in \Gamma r>0}\left(\frac{1}{r} \int_{\Gamma \cap O_{r}(z)} \omega(\xi)|d \xi|\right)\left(\frac{1}{r} \int_{\Gamma \cap O_{r}(z)}|\omega(\xi)|^{-\frac{1}{p-1}}|d \xi|\right)^{p-1}<+\infty .
$$

We will need some facts about the weights $\omega(\cdot)$, which satisfy the Muckenhoupt condition $A_{p}(\Gamma), 1 \leq p \leq+\infty$, on the rectifiable curve $\Gamma$. The following statement is true:

Statement 2.1. i) If $\omega \in A_{p}(\Gamma), 1 \leq p<+\infty$, then $\omega \in A_{q}(\Gamma)$, for $q>p$; ii) $\omega \in A_{p}(\Gamma), 1<p<+\infty$, if and only if $\omega^{-\frac{1}{p-1}} \in A_{p^{\prime}}(\Gamma), \frac{1}{p}+\frac{1}{p^{\prime}}=1$; iii) if $\omega \in A_{p}(\Gamma), 1<p<+\infty$, then $\omega \in A_{q}(\Gamma)$, for some $q: 1<q<p$.

For more information about this statement see, e.g., [16].

We will also use the following statement by R.R.Coifman, C.Fefferman [12].

Statement 2.2. If the function $\omega(\cdot)>0$ satisfies the Muckenhoupt condition $A_{p}(\Gamma), 1<p<+\infty$, then for sufficiently small $\delta>0$ the "inverse Hölder inequality"

$$
\left(\frac{1}{r} \int_{\Gamma \cap O_{r}(z)}|\omega(\xi)|^{1+\delta}|d \xi|\right)^{\frac{1}{1+\delta}} \leq c\left(\frac{1}{r} \int_{\Gamma \cap O_{r}(z)} \omega(\xi)|d \xi|\right), \forall r>0, \forall z \in \Gamma,
$$

holds, where $c=c(\delta)$ is a constant independent of $r$ and $z \in \Gamma$. 
As usual, by $L_{p, \omega}(\Gamma)$ we denote the weighted Lebesgue space of functions endowed with the norm $\|\cdot\|_{p, \omega}$ :

$$
\|f\|_{L_{p, \omega}(\Gamma)}=\left(\int_{\Gamma}|f(\xi)|^{p} \omega(\xi)|d \xi|\right)^{\frac{1}{p}} .
$$

Consider the singular Cauchy operator $S_{\Gamma}$ :

$$
S_{\Gamma}(f)=\frac{1}{2 \pi i} \int_{\Gamma} \frac{f(\xi)}{\xi-\tau} d \xi, \tau \in \Gamma
$$

The following theorem is valid (see, e.g. [15]).

Theorem D. Operator $S_{\Gamma}$ is bounded in $L_{p}(\Gamma), 1<p<+\infty$, only when $\Gamma$ is a regular curve. Moreover, if $\Gamma$ is a regular curve, then the singular operator $S_{\Gamma}$ is bounded in $L_{p, \omega}(\Gamma), 1<p<+\infty$, only when $\omega \in A_{p}(\Gamma)$.

Recall that the Lipschitz space $\operatorname{Lip}_{\alpha}(\Gamma)$ is a Banach space on $\Gamma$ with the norm

$$
\|f\|_{L_{i p_{\alpha}(\Gamma)}}=\max _{z \in \Gamma}|f(z)|+\sup _{z_{1} ; z_{2} \in \Gamma} \frac{\left|f\left(z_{1}\right)-f\left(z_{2}\right)\right|}{\left|z_{1}-z_{2}\right|^{\alpha}} .
$$

For more information about this result see, e.g., I.I.Danilyuk [14] and I.I.Privalov $[33]$.

Let $\Gamma \subset \mathbb{C}-$ be a rectifiable curve of length $l$ and $z=z(s), 0 \leq s \leq l$, its parametric equation with respect to the length of the arc $s . z(s)$ has a bounded change on $[0, l]$. Therefore, it has a finite derivative a.e. on $[0, l]$. Denote by $\theta(s), 0 \leq s \leq l$, the angle at the point $z=z(s)$ between the abscissa axis and the tangent (if there is) in the same point. If $\theta(s)$ has bounded variation, then the curve $\Gamma$ is called the Radon curve or a curve of bounded rotation If the function $\theta(s)$ satisfies the Holder condition with the order $\alpha \in(0,1]$, then $\Gamma$ is called a Lyapunov curve of order $\alpha$.

We will also use the following classical

Privalov theorem. The following statements hold true: i) Singular operator

$$
S_{\Gamma} f(z)=\frac{1}{2 \pi i} \int_{\Gamma} \frac{f(\xi)}{\xi-z} d \xi, z \in \Gamma,
$$

acts and is bounded in the space Lip $p_{\alpha}(\Gamma)$, if is a piecewise smooth curve with no cusps; ii) the same statement remains true when $\Gamma$ is a Radon curve with no cusps.

\section{Main assumptions. The weighted Smirnov classes. Some necessary facts}

Let $G(\xi)=|G(\xi)| e^{i \theta(\xi)}$ be complex-valued functions on the curve $\Gamma$. We make the following basic assumptions on the coefficient $G(\cdot)$ of the considered boundary value problem and $\Gamma$ :

(i) $G(\cdot)^{ \pm 1} \in L_{\infty}(\Gamma)$;

(ii) $\theta(\cdot)$ is piecewise continuous on $\Gamma$, and $\left\{\xi_{k}, k=\overline{1, r}\right\} \subset \Gamma$ are discontinuity points of the function $\theta(\cdot)$ :

We impose the following condition on the curve $\Gamma$.

(iii) $\Gamma$ is either Lyapunov or Radon curve (i.e. it is a limited rotation curve) with no cusps. Direction along $\Gamma$ will be considered as positive, i.e. when moving 
along this direction the domain $D$ stays on the left side. Let $a \in \Gamma$ be an initial (and also a final) point of the curve $\Gamma$. We will assume that $\xi \in \Gamma$ follows the point $\tau \in \Gamma$, i.e. $\tau \prec \xi$, if $\xi$ follows $\tau$ when moving along a positive direction on $\Gamma \backslash a$, where $a \in \Gamma$ represents two stuck points $a^{+}=a^{-}$, with $a^{+}$a beginning, and $a^{-}$an end of the curve $\Gamma$.

So, without loss of generality, we will assume that $a^{+} \prec \xi_{1} \prec \ldots \prec \xi_{r} \prec b=a^{-}$. Denote one-sided $\operatorname{limits}_{\substack{\xi \rightarrow \xi_{0} \pm 0 \\ \xi \in \Gamma}} g(\xi)$ of the function $g(\xi)$ at the point $\xi_{0} \in \Gamma$ generated by this order by $g\left(\xi_{0} \pm 0\right)$, respectively. The jumps of the function $\theta(\xi)$ at the points $\xi_{k}, k=\overline{1, r}$, are denoted by $h_{k}: h_{k}=\theta\left(\xi_{k}+0\right)-\theta\left(\xi_{k}-0\right)$, $k=\overline{1, r}$.

Let $D^{+} \subset \mathbb{C}$ be a bounded domain with the boundary $\Gamma=\partial D^{+}$, which satisfies the condition iii). Denote by $E_{p}\left(D^{+}\right), 1<p<\infty$, a Smirnov Banach space of analytic functions in $D^{+}$with the norm $\|\cdot\|_{E_{p}\left(D^{+}\right)}$:

$$
\|f\|_{E_{p}\left(D^{+}\right)}=:\left\|f^{+}\right\|_{L_{p}(\Gamma)}, \forall f \in E_{p}\left(D^{+}\right),
$$

where $f^{+}=\left.f\right|_{\Gamma}$ are non-tangential boundary values of the function $f$ on $\Gamma$.

Based on the norm (3.1), we define the weighted Smirnov class. Let $\rho \in L_{1}(\Gamma)$ be some weight function. Define weighted Smirnov class $E_{p, \rho}\left(D^{+}\right)$:

$$
E_{p, \rho}\left(D^{+}\right) \equiv\left\{f \in E_{1}\left(D^{+}\right):\left\|f^{+}\right\|_{L_{p, \rho}(\Gamma)}<+\infty\right\},
$$

and let

$$
\|f\|_{E_{p, \rho}\left(D^{+}\right)}=\left\|f^{+}\right\|_{L_{p, \rho}(\Gamma)} .
$$

Similarly we define the Smirnov classes in unbounded domain. Let $D^{-} \subset$ $\mathbb{C}$ be an unbounded domain containing infinitely remote point $(\infty)$. Denote by ${ }_{m} E_{1}\left(D^{-}\right)$a class of functions from $E_{1}\left(D^{-}\right)$which are analytic in $D^{-}$and have an order $k \leq m$ at infinity, i.e. the function $f \in E_{1}\left(D^{-}\right)$has a Laurent decomposition $f(z)=\sum_{k=-\infty}^{m} a_{k} z^{k}$ in the vicinity of the infinitely remote point $z=\infty$, where $m$ is some integer.

For a given weight function $\rho \in L_{1}(\Gamma)$, the weighted class ${ }_{m} E_{p, \rho}\left(D^{-}\right)$is defined as follows

with

$$
{ }_{m} E_{p, \rho}\left(D^{-}\right) \equiv\left\{f \in_{m} E_{1}\left(D^{-}\right):\left\|f^{-}\right\|_{L_{p, \rho}(\Gamma)}\right\},
$$

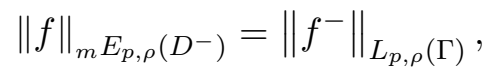

where $f^{-}$are non-tangential boundary values of the function $f$ on $\Gamma$.

We will also use some results of [35] related to the solvability of homogeneous Riemann problem in weighted Smirnov classes. Consider the following homogeneous Riemann problem in weighted Smirnov classes

$$
F^{+}(\xi)+G(\xi) F^{-}(\xi)=0, \text { a.e. } \xi \in \Gamma .
$$

By the solution of the problem (3.2) we mean a pair of analytic functions

$$
\left(F^{+} ; F^{-}\right) \in E_{p, \rho}\left(D^{+}\right) \times_{m} E_{p, \rho}\left(D^{-}\right),
$$

whose non-tangential boundary values $F^{ \pm}(\xi)$ satisfy the equality (3.2) a.e. on $\Gamma$. In weightless case, this problem has been well enough studied in the monograph by I.I.Danilyuk [14]. 
Let $S$ be a length of the curve $\Gamma$ and $z=z(s), 0 \leq s \leq S$, be a parametric representation of $\Gamma$ with respect to the length of the arc $s$. Rewrite the problem (3.2) as follows

$$
F^{+}[z(s)]+G(z(s)) F^{-}[z(s)]=0, \text { a.e. } s \in[0, S],
$$

Let $\Omega(s) \equiv \theta(z(s)), 0 \leq s \leq S$, and suppose

$$
h_{k}=\Omega\left(s_{k}+0\right)-\Omega\left(s_{k}-0\right), k=\overline{1, r} ; h_{0}=\Omega(+0)-\Omega(S-0),
$$

where $\xi_{k}=z\left(s_{k}\right), 0<s_{k}<S, a=z(0)=z(S)$, are discontinuity points of the argument $\Omega(\cdot)$. Consider the following piecewise holomorphic functions

$$
\begin{gathered}
Z_{(1)}(z)=\exp \left\{\frac{1}{2 \pi i} \int_{\Gamma} \ln |G(z(s))| \frac{d z(s)}{z(s)-z}\right\}, \\
\tilde{Z}_{\theta}(z)=\exp \left\{\frac{1}{2 \pi} \int_{\Gamma} \Omega(s) \frac{d z(s)}{z(s)-z}\right\}=\exp \left\{\frac{1}{2 \pi} \int_{\Gamma} \theta(z(s)) \frac{d z(s)}{z(s)-z}\right\}, z \notin \Gamma .
\end{gathered}
$$

As the argument $\theta(\cdot)$ is defined ambiguously, it is clear that the value of the function $\tilde{Z}_{\theta}(\cdot)$ depends on the chosen argument. Denote the product of these functions by

$$
Z_{\theta}: Z_{\theta}(z) \equiv Z_{(1)}(z) \tilde{Z}_{\theta}(z), z \in \mathbb{C} \backslash \Gamma .
$$

Hereinafter, the function $Z_{\theta}(\cdot)$ will be called a canonical solution of the problem (3.2) corresponding to the argument $\theta(\cdot)$.

The following lemma is true for the first multiplier $Z_{(1)}(z)$.

Lemma 3.1. [14] Let the conditions $i$ )-iii be satisfied for the coefficient $G(\cdot)$ and the curve $\Gamma$. Then the functions $Z_{(1)}(z) ; Z_{(1)}^{-1}(z)$ are bounded in each of the domains $D^{ \pm}$.

To proceed further, we represent the function $\Omega(s)$ in the following form

$$
\Omega(s)=\Omega_{0}(s)+\Omega_{1}(s), 0 \leq s \leq S,
$$

where $\Omega_{0}(s)$ is a continuous part, and $\Omega_{1}(s)$ is a jump function defined by

$$
\begin{gathered}
\Omega_{1}(0)=0, \\
\Omega_{1}(s)=[\Omega(+0)-\Omega(0)]+\sum_{0<s_{k}<s} h_{k}+[\Omega(s)-\Omega(s-0)], 0<s<S .
\end{gathered}
$$

Denote

$$
h_{0}^{(0)}=\Omega_{0}(S)-\Omega_{0}(0), \quad h_{0}^{(1)}=\Omega_{1}(+0)-\Omega_{1}(S-0) .
$$

Let

and

$$
Z_{(2)}(z)=\exp \left\{\frac{1}{2 \pi} \int_{\Gamma} \Omega_{0}(s) \frac{d z(s)}{z(s)-z}\right\}
$$

$$
Z_{(3)}(z)=\exp \left\{\frac{1}{2 \pi} \int_{\Gamma} \Omega_{1}(s) \frac{d z(s)}{z(s)-z}\right\} .
$$

It was proved in [8] that the following inclusion is true

$$
\tilde{Z}_{(2)}^{ \pm 1}(s)=|z(s)-z(0)|^{ \pm \frac{h_{0}^{(0)}}{2 \pi}}\left|Z_{(2)}^{ \pm}[z(s)]\right|^{ \pm 1} \in L_{q}(\Gamma), \forall q \in(0,+\infty) .
$$


The modulus of boundary values of the function $Z_{(3)}(\cdot)$ can be represented as follows [8]:

$$
\left|Z_{(3)}^{+}[z(\sigma)]\right| \equiv|z(0)-z(\sigma)|^{-\frac{h_{0}^{(1)}}{2 \pi}} \prod_{0<s_{k}<S}\left|z\left(s_{k}\right)-z(\sigma)\right|^{-\frac{h_{k}}{2 \pi}},
$$

which follows directly from the lemma below.

Lemma 3.2. [14] Let the curve $\Gamma$ satisfy the condition iii) and $\Omega_{1}(s)$ be an arbitrary jump function with the jumps $h_{0}^{(1)}=\Omega_{1}(+0)-\Omega_{1}(S-0)$ at the point $z(0)$. Then the modulus of boundary values of the function $Z_{(3)}(\cdot)$ can be represented by the formula (3.4) for a.e. $\sigma \in[0, S]$

The modulus of boundary values $\left|Z_{\theta}^{+}(z(s))\right|$ can be represented as follows

$$
\left|Z_{\theta}^{+}(z(s))\right| \equiv\left|Z_{(1)}^{+}(z(s))\right|\left|\tilde{Z}_{(2)}(s)\right| \sigma(s) .
$$

From Lemma 3.1 we have

$$
\left|Z_{\theta}^{+}(z(s))\right| \sim \sigma(s)\left|\tilde{Z}_{(2)}(s)\right|, s \in[0, S] .
$$

\section{Main result}

Consider the nonhomogeneous Riemann problem

$$
F^{+}(z(s))-G(z(s)) F^{-}(z(s))=g(z(s)), s \in(0, S),
$$

where $g \in L_{p, \rho}(\Gamma)$ is a given function. By the solution of the problem (4.1) we mean a pair of functions

$$
\left(F^{+}(z) ; F^{-}(z)\right) \in E_{p, \rho}\left(D^{+}\right) \times_{m} E_{p, \rho}\left(D^{-}\right),
$$

whose boundary values $F^{ \pm}$on $\Gamma$ a.e. satisfy (4.1). Consider the following Cauchy type integral

$$
F_{1}(z) \equiv \frac{Z_{\theta}(z)}{2 \pi i} \int_{\Gamma} \frac{g(\xi) d \xi}{Z_{\theta}^{+}(\xi)(\xi-z)}
$$

and let

$$
\sigma(s) \equiv|z(0)-z(s)|^{-\frac{h_{0}}{2 \pi}} \prod_{0<s_{k}<S}\left|z\left(s_{k}\right)-z(s)\right|^{-\frac{h_{k}}{2 \pi}},
$$

where $h_{0}=h_{0}^{(1)}-h_{0}^{(0)}$.

Introduce the following weight function

$$
\nu(s)=: \sigma^{p}(s) \rho(z(s)), s \in(0, S) .
$$

We will assume that the weight function $\rho(\cdot)$ satisfies the condition

$$
\int_{\Gamma} \rho^{-\frac{q}{p}}(z(s))|d z(s)|<+\infty .
$$

The following main theorem is true. 
Theorem 4.1. Let the coefficient $G(z(s)), 0 \leq s \leq S$, of the problem (4.1) satisfy the conditions (i), (ii), its argument be a piecewise Hölder function and the curve $\Gamma=z([0, S])$ satisfy the condition (iii). Let the weight function $\nu(\cdot)$ defined by (4.3) belong to the Muckenhoupt class $A_{p}(\Gamma), 1<p<+\infty$, and the weight function $\rho(\cdot)$ satisfy the condition (4.4). Then the analytic function $F(\cdot)$ defined by the Cauchy integral (4.2) is a particular solution of nonhomogeneous Riemann problem (4.1) in weighted Smirnov classes $E_{p ; \rho}\left(D^{+}\right) \times_{-1} E_{p ; \rho}\left(D^{-}\right)$.

Proof. Applying Sokhotski-Plemelj formulas to (4.2), we obtain that the boundary values $F^{ \pm}(\xi)$ satisfy the relation (4.1) a.e. on $\Gamma$. Let's show that the function $F(z)$ belongs to the required classes. Sokhotski-Plemelj formulas yield

$$
F^{+}(\xi)=\frac{1}{2} g(\xi)+\frac{Z_{\theta}^{+}(\xi)}{2 \pi i} \int_{\Gamma} \frac{g(\tau) d \tau}{Z_{\theta}^{+}(\xi)(\tau-\xi)}, \xi \in \Gamma .
$$

Denote

We have

$$
f(\xi) \equiv g(\xi)\left[Z_{\theta}^{+}(\xi)\right]^{-1}, \xi \in \Gamma \text {. }
$$

$$
\Phi(\xi)=\frac{1}{2} f(\xi)+\frac{1}{2 \pi i} \int_{\Gamma} \frac{f(\tau) d \tau}{\tau-\xi},
$$

where

$$
\Phi(\xi)=F^{+}(\xi)\left[Z_{\theta}^{+}(\xi)\right]^{-1} \text {. }
$$

It is not difficult to see that the function $f(\cdot)$ belongs to the space $L_{p ; \mu}(\Gamma)$ if and only if the function $g(\cdot)$ belongs to the space $L_{p ; \rho}(\Gamma)$, where the weight function $\mu(\cdot)$ is defined by

$$
\mu(s)=\left|Z_{\theta}^{+}(z(s))\right|^{p} \rho(z(s)), s \in(0, S) .
$$

In the sequel, we will assume that the weight $\nu(\cdot)$ satisfies the Muckenhoupt condition $A_{p}(\Gamma)$. Statement 2.1 implies the inclusion $\nu(\cdot) \in L_{1}(\Gamma)$. Moreover, $\exists p_{3}>1: \nu^{p_{3}}(\cdot) \in L_{1}(\Gamma)$. Then, after applying Hölder's inequality we obtain

$$
\int_{\Gamma} \nu(s)\left|\tilde{Z}_{(2)}(s)\right|^{p}|d z(s)| \leq\left(\int_{\Gamma} \nu^{p_{3}}(s)|d z(s)|\right)^{\frac{1}{p_{3}}}\left(\int_{\Gamma}\left|\tilde{Z}_{(2)}(s)\right|^{p q_{3}}|d z(s)|\right)^{\frac{1}{q_{3}}},
$$

where $\frac{1}{p_{3}}+\frac{1}{q_{3}}=1$. Taking into account (3.3), we immediately obtain

$$
\int_{\Gamma} \nu(s)\left|\tilde{Z}_{(2)}(s)\right|^{p}|d z(s)|<+\infty .
$$

Let's show that there exists $r \in(1, p)$ such that the function $\Phi(\cdot)$ belongs to Smirnov class $E_{r}\left(D^{ \pm}\right)$. To do so, it suffices to show that $f(\cdot) \in L_{r}(\Gamma)$. The rest will follow from Theorem D. As $\nu(\cdot)$ belongs to the class $A_{p}(\Gamma)$, from Statement 2.1 it follows that $\exists q \in(1, p): \nu(\cdot) \in A_{q}(\Gamma)$. Let $r=\frac{p}{q}$. We have

$$
\begin{gathered}
\int_{\Gamma}|f|^{r}|d z(s)|=\int_{\Gamma}|f|^{r} \mu^{\frac{1}{q}}(s) \mu^{-\frac{1}{q}}(s)|d z(s)| \leq \\
\leq\left(\int_{\Gamma}|f|^{p} \mu(s)|d z(s)|\right)^{\frac{1}{q}}\left(\int_{\Gamma} \mu^{-\frac{q^{\prime}}{q}}(s)|d z(s)|\right)^{\frac{1}{q^{\prime}}},
\end{gathered}
$$

where $\frac{1}{q}+\frac{1}{q^{\prime}}=1$. Considering (3.5), for $\mu(s)$ we obtain

$$
\mu(s) \sim \sigma^{p}(s)\left|\tilde{Z}_{(2)}^{+}(s)\right|^{p} \rho(z(s))=\nu(s)\left|\tilde{Z}_{(2)}^{+}(s)\right|^{p}, s \in(0, S) .
$$


Let's show that

$$
\int_{\Gamma} \mu^{-\frac{q^{\prime}}{q}}(s)|d z(s)|<+\infty
$$

i.e.

$$
\int_{\Gamma} \nu^{-\frac{q^{\prime}}{q}}(s)\left|\tilde{Z}_{(2)}^{+}(s)\right|^{-\frac{q^{\prime}}{q} p}|d z(s)|<+\infty .
$$

From Statement 2.1 it follows that $\nu(\cdot) \in A_{q}(\Gamma) \Leftrightarrow \nu^{-\frac{1}{q-1}}(\cdot) \in A_{q^{\prime}}(\Gamma)$. For simplicity, we let $\eta(s)=\nu^{-\frac{1}{q-1}}(s)$. Let $\delta>0$ be a sufficiently small number and $q_{0}=1+\delta$. Then, in view of $-\frac{q^{\prime}}{q}=-\frac{1}{q-1}$ and applying Hölder's inequality, from (4.9) we obtain $\left(\gamma=-\frac{q^{\prime}}{q} p\right)$ :

$$
\int_{\Gamma} \eta(s)\left|\tilde{Z}_{(2)}(s)\right|^{\gamma}|d z(s)| \leq\left(\int_{\Gamma} \eta^{q_{0}}(s)|d z(s)|\right)^{\frac{1}{q_{0}}}\left(\int_{\Gamma}\left|\tilde{Z}_{(2)}(s)\right|^{\gamma q_{0}^{\prime}}|d z(s)|\right)^{\frac{1}{q_{0}^{\prime}}}
$$

where $\frac{1}{q_{0}}+\frac{1}{q_{0}^{\prime}}=1$. From $\eta(\cdot) \in A_{q_{0}}(\Gamma)$ it follows that $\eta(\cdot) \in L_{q_{0}}(\Gamma)$. Taking into account the relation (3.3), from (4.10) and Statement 2.2 (inverse Hölder inequality) we obtain (4.9). As $f \in L_{p ; \mu}(\Gamma)$, it follows from (4.10) that $f \in L_{r}(\Gamma)$ for some $r \in(1, p)$. Then, as is known (see, e.g., [14]), the function $\Phi(\cdot)$ belongs to Smirnov classes $E_{r}\left(D^{ \pm}\right)$. As $Z_{\theta}(\cdot) \in E_{\delta}\left(D^{ \pm}\right)$for sufficiently small $\delta>0$, then, applying Hölder's inequality, from (4.2) we obtain that the function $F(\cdot)$ belongs to Smirnov classes $E_{\delta}\left(D^{ \pm}\right)$for sufficiently small $\delta>0$. It remains to prove that the function $F(\cdot)$ belongs to the required Smirnov classes, i.e. to classes $E_{p ; \rho}\left(D^{+}\right) \times_{m} E_{p ; \rho}\left(D^{-}\right)$. By definition of these classes, to do so we need to show that $F_{1}(\cdot) \in E_{1}\left(D^{+}\right) \times_{m} E_{1}\left(D^{-}\right)$and $F_{1}^{ \pm}(\cdot) \in L_{p ; \rho}(\Gamma)$.

In what follows, we will assume that the argument $\theta(\cdot)$ of the coefficient $G(\cdot)$ is a piecewise Hölder function on the curve $\Gamma$. Then it is obvious that the continuous part $\Omega_{0}(s)$ of the function $\Omega(s)=\theta(z(s)), s \in[0, S]$, is a Hölder function on $[0, S]$. In this case, it follows from the results obtained in [8] that the function $\tilde{Z}_{(2)}(\cdot)$ is a Hölder function on $[0, S]$ and neither vanishes nor becomes infinite, i.e.

$$
\max _{[0, S]}\left|\tilde{Z}_{(2)}(\cdot)\right|^{ \pm 1}<+\infty \text {. }
$$

In fact, let $h_{0}^{(0)}=\Omega_{0}(S)-\Omega_{0}(0)$. By Privalov theorem, the singular integral

$$
\int_{0}^{S} \Omega_{0}(s) \operatorname{Re} \frac{d z(s)}{z(s)-z(\sigma)}
$$

is a function which is continuous in the sense of Hölder at every internal point $\sigma$ : $0<\sigma<S$. To study its behavior in a neighborhood of the point $\sigma=0(\bmod S)$, we redefine the function $\Omega_{0}(s)$ at the point $s=0$ by letting $\Omega_{0}(0)=\Omega_{0}(S)$ and extend the obtained function periodically (with a period $S$ ) onto all real $s$ 's. If we now introduce the jump function $\Omega_{0_{1}}(s)$ of the obtained $\Omega_{0}(s)$ on the segment $\left[-\frac{S}{2}, \frac{S}{2}\right]$, then the function $\Omega_{0}(s)-\Omega_{0_{1}}(s)$ will be defined on $\left[-\frac{S}{2}, \frac{S}{2}\right]$ and continuous in the sense of Hölder in a neighborhood of the point $s=0$. Quite similarly, we extend the function $z(s)$ onto the whole real axis periodically (with 
a period $S$ ) and denote the obtained function again by $z(\cdot)$. So we have

$$
\begin{gathered}
\int_{0}^{S} \Omega_{0}(s) \operatorname{Re} \frac{d z(s)}{z(s)-z(\sigma)}=\int_{-S / 2}^{S / 2} \Omega_{0}(s) \operatorname{Re} \frac{d z(s)}{z(s)-z(\sigma)}= \\
=\int_{-S / 2}^{S / 2}\left[\Omega_{0}(s)-\Omega_{0_{1}}(s)\right] \operatorname{Re} \frac{d z(s)}{z(s)-z(\sigma)}+\int_{-S / 2}^{S / 2} \Omega_{0_{1}}(s) \operatorname{Re} \frac{d z(s)}{z(s)-z(\sigma)}= \\
=J_{1}(\sigma)+J_{2}(\sigma) .
\end{gathered}
$$

In view of

$$
\operatorname{Re} \frac{d z(s)}{z(s)-z(\sigma)}=d_{s} \ln |z(s)-z(\sigma)|,
$$

and applying the integration by parts formula, we obtain

$$
\begin{gathered}
I_{2}(\sigma)=\int_{-S / 2}^{S / 2} \Omega_{0_{1}}(s) \operatorname{Re} \frac{d z(s)}{z(s)-z(\sigma)}=\int_{-S / 2}^{S / 2} \Omega_{0_{1}}(s) d_{s} \ln |z(s)-z(\sigma)|= \\
=\Omega_{0_{1}}(S / 2) \ln |z(S / 2)-z(\sigma)|-\Omega_{0_{1}}(-S / 2) \ln |z(-S / 2)-z(\sigma)|- \\
=\int_{-S / 2}^{S / 2} \ln |z(s)-z(\sigma)| d \Omega_{0_{1}}(s) .
\end{gathered}
$$

Taking into account that $\Omega_{0_{1}}(-S / 2)-\Omega_{0_{1}}(S / 2)=h_{0}^{(0)}$ and $z(s)$ is a periodic function with a period $S$, we get

$$
I_{2}(\sigma)=-\int_{-S / 2}^{S / 2} \ln |z(s)-z(\sigma)| d \Omega_{0_{1}}(s)+h_{0}^{(0)} \ln |z(S / 2)-z(\sigma)| .
$$

Let's calculate the integral on the right-hand side of the last relation. Let $\sigma \in$ $\left(-\frac{S}{2}, \frac{S}{2}\right) \backslash\{0\}$ be an arbitrary number. We have

$$
\begin{gathered}
I_{3}(\sigma)=\int_{-S / 2}^{S / 2} \ln |z(s)-z(\sigma)| d \Omega_{0_{1}}(s)= \\
=\lim _{\varepsilon \rightarrow+0}\left(\int_{-S / 2}^{\sigma-\varepsilon} \ln |z(s)-z(\sigma)| d \Omega_{0_{1}}(s)+\int_{\sigma+\varepsilon}^{S / 2} \ln |z(s)-z(\sigma)| d \Omega_{0_{1}}(s)\right) .
\end{gathered}
$$

Without loss of generality, we will assume that $\sigma \in\left(-\frac{S}{2}, 0\right)$ (the case of $\sigma \in\left(0, \frac{S}{2}\right)$ is treated similarly). As the function $\Omega_{0_{1}}(s)$ is constant on $\left(-\frac{S}{2}, 0\right) \cup\left(0, \frac{S}{2}\right)$, for sufficiently small $\varepsilon>0$ we obtain

$$
\begin{gathered}
I_{3}(\sigma)=\lim _{\varepsilon \rightarrow+0} \int_{\sigma+\varepsilon}^{S / 2} \ln |z(s)-z(\sigma)| d \Omega_{0_{1}}(s)= \\
=\lim _{\varepsilon \rightarrow+0}\left[\left(\Omega_{0_{1}}(\sigma+\varepsilon+0)-\Omega_{0_{1}}(\sigma+\varepsilon)\right) \ln |z(\sigma+\varepsilon)-z(\sigma)|\right]+ \\
+\left(\Omega_{0_{1}}\left(\frac{S}{2}\right)-\Omega_{0_{1}}\left(\frac{S}{2}-0\right)\right) \ln \left|z\left(\frac{S}{2}\right)-z(\sigma)\right|-h_{0}^{(0)} \ln |z(0)-z(\sigma)| .
\end{gathered}
$$

As the function $\Omega_{0_{1}}(s)$ is continuous at the point $\sigma \in\left(-\frac{S}{2}, 0\right)$ (we assume that it is continuous from the left), it follows from the previous relation that

$$
I_{3}(\sigma)=\ln |z(0)-z(\sigma)|^{-h_{0}^{(0)}}
$$


So we ultimately obtain

$$
\begin{gathered}
\int_{0}^{S} \Omega_{0}(s) \operatorname{Re} \frac{d z(s)}{z(s)-z(\sigma)}=\int_{-S / 2}^{S / 2}\left[\Omega_{0}(s)-\Omega_{0_{1}}(s)\right] \operatorname{Re} \frac{d z(s)}{z(s)-z(\sigma)}+ \\
+h_{0}^{(0)} \ln |z(S / 2)-z(\sigma)|-h_{0}^{(0)} \ln |z(\sigma)-z(0)|
\end{gathered}
$$

As the first integral on the right-hand side is a Hölder function in a neighborhood of the point $\sigma=0$ (due to Privalov theorem), it directly follows that the functions

$$
\left|\tilde{Z}_{(2)}(\sigma)\right|^{\mp 1}=|z(\sigma)-z(0)|^{\mp_{2 \pi}^{h_{0}^{(0)}}} \exp \left\{\mp \frac{1}{2 \pi} \int_{0}^{S} \Omega_{0}(s) \operatorname{Re} \frac{d z(s)}{z(s)-z(\sigma)}\right\},
$$

are bounded in a neighborhood of the point $\sigma=0(\bmod S)$, and, consequently, on the whole interval $[0, S]$. Thus, the relation $(4.11)$ is true. Then from $(3.5)$ we obtain

$$
\left|Z_{\theta}^{+}(z(s))\right| \sim \sigma(s), s \in[0, S] .
$$

As a result, we get

$$
\nu(s) \sim \mu(s), s \in[0, S] .
$$

As $\nu(\cdot) \in A_{p}(\Gamma), 1<p<+\infty$, it is clear that $\mu(\cdot) \in A_{p}(\Gamma)$. Then from Theorem $\mathrm{D}$ and (4.4) it follows that the function $\Phi(\cdot)$ belongs to the space $L_{p ; \mu}(\Gamma)$, because $f \in L_{p ; \mu}(\Gamma)$. We have

$$
\begin{gathered}
\int_{\Gamma}|\Phi(z(s))|^{p} \mu(s)|d z(s)|=\int_{\Gamma}\left|F^{+}(z(s))\right|^{p}\left|Z^{+}(z(s))\right|^{-p} \mu(s)|d z(s)|= \\
=\int_{\Gamma}\left|F^{+}(z(s))\right|^{p} \rho(z(s))|d z(s)|<+\infty .
\end{gathered}
$$

Consequently, $F^{+}(\cdot) \in L_{p ; \rho}(\Gamma)$. Applying Hölder's inequality, we obtain

$$
\begin{gathered}
\int_{\Gamma}\left|F^{+}(z(s))\right||d z(s)| \leq \\
\leq\left(\int_{\Gamma}\left|F^{+}(z(s))\right|^{p} \rho(z(s))|d z(s)|\right)^{\frac{1}{p}}\left(\int_{\Gamma} \rho^{-\frac{q}{p}}(z(s))|d z(s)|\right)^{\frac{1}{q}} .
\end{gathered}
$$

Then it follows from (4.4) that $F^{+}(\cdot) \in L_{1}(\Gamma)$ and, as a result, by Smirnov theorem we have the inclusion $F(\cdot) \in E_{1}\left(D^{ \pm}\right)$.

The theorem is proved.

\section{Acknowledgements}

The author would like to express her deepest gratitude to Professor B.T. Bilalov for his encouragement and valuable guidance throughout this research.

The author thanks the reviewer for his/her careful reading of the manuscript and constructive remarks. 


\section{References}

[1] D.R. Adams, Morrey Spaces, Springer, Switzerland, 2016.

[2] B.T. Bilalov, Basicity of some exponential, sine and cosine systems, Difer. Uravn. 26(1) (1990), 10-16. (in Russian)

[3] B.T. Bilalov, Basis properties of some exponential, sine and cosine systems, Sib. Math. J. 45(2) (2004), 264-273. (in Russian)

[4] B.T. Bilalov, On basicity of the system and exponential shift systems, Dokl. RAN 345(2) (1995), 644-647. (in Russian)

[5] B.T. Bilalov, Basis properties of power systems in $L_{p}$, Sib. Math. J. 47(1) (2006), 1-12. (in Russian)

[6] B.T. Bilalov, Exponential shift system and the Kostyuchenko problem, Sib. Math. J. 50(2) (2009), 279-288. (in Russian)

[7] B.T. Bilalov, On solution of the Kostyuchenko problem, Sib. Math. J. 53(3) (2012), 509-526.

[8] B.T. Bilalov, The basis property of a perturbed system of exponentials in Morreytype spaces, Sib. Math. J. 60(2) (2019), 323-350.

[9] B.T. Bilalov, T.B. Gasymov, A.A. Guliyeva, On solvability of Riemann boundary value problem in Morrey-Hardy classes, Turkish J. Math. 40(5) (2016), 1085-1101.

[10] A.V. Bitsadze, On one system of functions, Uspekhi Mat. Nauk 5(4(38)) (1950), 150-151 (in Russian)

[11] T. Carleman, Sur la théorie des equations intégrales et ses applications, Verband des Int. math. Kongr., Zurich, 1, 1932.

[12] R.R. Coifman, C. Fefferman, Weighted norm inequalities for maximal functions and singular integrals, Studia Math. 51(3) (1974), 241-249.

[13] D.V. Cruz-Uribe, A. Fiorenza, Variable Lebesgue spaces: Foundations and Harmonic Analysis, Springer, 2013.

[14] I.I. Danilyuk, Irregular boundary value problems on half plane, Moscow, Nauka, 1975, 256 p. (in Russian)

[15] G. David, Operateur sintegraux singulars sur certain scourbes du plan complexe, Ann. Sci. Ecole Norm. Sup. 17 (1984), 157-189.

[16] E.M. Dynkin, Methods of theory of singular integrals, Itoginauki i texn., ser. matem. analiz 15 (1987), 197-292. (in Russian)

[17] F.D. Gakhov, Boundary value problems, Moscow, Nauka, 1977, 640 p. (in Russian)

[18] D. Hilbert, Über eine Anwendung der Integral der Integralgleichungen auf ein Problem der Fuktionentheorie, Verhand 3 I. Math. Kong, Heidelberg, 1904.

[19] D. Hilbert, Grundzüge einer allgemeinen Theorie der linearen Integralgleichungen, Teubner, Leipzig and Berlin, 1912.

[20] D.M. Israfilov, N.P. Tozman, Approximation in Morrey-Smirnov classes, Azerb. J. Math. 1(1) (2011), 99-113.

[21] V.M. Kokolashvili, A. Meskhi, H. Rafeiro, S. Samko, Integral operators in nonstandart function spaces, Birkhauser, 2010.

[22] V.M. Kokilashvili, Boundary value problems of analytic and harmonic functions in a domain with piecewise smooth boundary in the frame of variable exponent Lebesgue spaces, Operator Theory 216 (2011), 17-39.

[23] G. Litvinchuk, Boundary-Value Problems and Singular Integral Equations with Shift, Moscow, Nauka, 1977. (in Russian)

[24] G. Manjavidze, N. Manjavidze, Boundary value problems for analytic and generalized analytic functions, J. Math. Sci. 160 (2009), 745-821.

[25] Z. Meshveliani, The Riemann-Hilbert problem in weighted Smirnov classes of analytic functions, Proc. Razmadze Math. Inst. 137 (2005), 65-86. 
[26] E.I. Moiseev, On basicity of sine and cosine systems, Dokl. Akad. Nauk SSSR 275(4) (1984), 794-798. (in Russian)

[27] E.I. Moiseev, On basicity of one sine system, Differ. Uravn. 23(1) (1987), 177-179. (in Russian)

[28] N. Muskhelishvili, Singular Integral Equations, Noordhoff, Groningen-Leyden, 1953.

[29] T.I. Najafov, N.P. Nasibova, On the Noetherness of the Riemann problem in a generalized weighted Hardy classes, Azerb. J. Math. 5(2) (2015), 109-139.

[30] J. Plemelj, Riemannsche Functionenscharen mit gegebener Monodromiargruppe, Monatsh. Math. Phys. 19 (1908), 211-245.

[31] S.M. Ponomarev, On an eigenvalue problem, Dokl. Akad. Nauk SSSR 249(5) (1979), 1068-1070 (in Russian)

[32] S.M. Ponomarev, On the theory of boundary value problems for mixed type equations in three-dimensional domains, Dokl. Akad. Nauk SSSR 246(6) (1979), 13031304. (in Russian)

[33] I. Privalov, Boundary Properties of Analytic Functions, Moscow, Gostekhizdat, 1950. (in Russian)

[34] B. Riemann, Grundlagen für eine allgemeine Theorie der. Functionen einer veränderlichen complexen Grösse, Göttingen, 1851; Werke, Leipzig, 1876.

[35] S.R. Sadigova, The general solution of the homogeneous Riemann problem in the weighted Smirnov classes, Proc. Inst. Math. Mech. Natl. Acad. Sci. Azerb. 40(2) (2014), 115-124.

[36] S.R. Sadigova and A.E. Guliyeva, On the solvability of Riemann problem in the weighted Smirnov classes, Anal. Math. 44(4) (2018), 587-603.

[37] I.N. Vekua, New Methods of the Solutions of Elliptic Equations, Moscow, Gostekhizdat, 1948. (in Russian)

[38] I.N. Vekua, Generalized Analytic Functions, Pergamon Press, Oxford, 1962.

[39] I.N. Vekua, Systems of Singular Integral Equations, Moscow, Nauka, 1970. (in Russian)

Sabina R. Sadigova

Institute of Mathematics and Mechanics of NAS of Azerbaijan, Baku, Azerbaijan.

E-mail address: s_sadigova@mail.ru

Received: February 19, 2020; Revised: July 21, 2020; Accepted: September 3, 2020 